\title{
Effect of modified compound calcium phosphate cement on the differentiation and osteogenesis of bone mesenchymal stem cells
}

\author{
Jican Zeng ${ }^{1}$, Jiazhong Lin ${ }^{1,2}$, Guanfeng Yao ${ }^{1}$, Kangmei Kong ${ }^{1}$ and Xinjia Wang ${ }^{1 *}$
}

\begin{abstract}
Background: The aim of this study is to evaluate the effect of self-invented compound calcium phosphate cement upon the proliferation and osteogenesis of bone mesenchymal stem cells (BMSCs).

Methods: Four groups including traditional calcium phosphate cement, modified calcium phosphate cement, modified calcium phosphate cement plus bone morphogenetic protein (BMP), and control groups were established. The cell proliferation curve was delineated by MTT. The activity of BMSCs to synthesize alkaline phosphatase (AKP) was evaluated. The growth and invasion of BMSCs were observed. The expression levels of aggrecan, collagen I, collagen II, AKP, and OSX messenger RNA (mRNA) were measured by using RT-PCR.

Results: Compared with other groups, the BMSCs in the modified calcium phosphate cement group presented with loose microstructure and the BMSCs closely attached to the vector margin. At 7 days after co-culture, the expression of AKP in the modified calcium phosphate cement plus BMP group was significantly upregulated compared with those in other groups. In the modified calcium phosphate cement group, the BMSCs properly proliferated on the surface of bone cement and invaded into the cement space. At 10 days, the expression levels of aggrecan, collagen I, collagen II, AKP, and OSX mRNA in the modified calcium phosphate cement and modified calcium phosphate cement plus BMP groups were significantly upregulated than those in other groups.

Conclusions: Modified compound calcium phosphate cement possesses excellent biocompatibility and osteogenic induction ability. Loose microstructure and large pore size create a favorable environment for BMSCs proliferation and vascular invasion, as an ideal vector for releasing BMP cytokines to mediate the differentiation and osteogenesis of BMSCs.
\end{abstract}

Keywords: Bone cement, Differentiation induction, Bone morphogenetic protein

\section{Background}

Calcium phosphate cement is a bioactive and biodegradable grafting material in the form of powder and liquid, which, when mixed, sets as primarily hydroxyapatite, occasionally mingled with unreacted particles and other phases. This material has been extensively investigated due to its excellent biomechanical property, potential resorbability, molding capability, and convenient manipulation. Because

\footnotetext{
* Correspondence: xinjiangwang2017@sina.com

'Department of Spine Surgery, The Second Affiliated Hospital to Shantou University Medical College, The DongXia North Road, Shantou 515041, Guangdong, China

Full list of author information is available at the end of the article
}

calcium phosphate cement can potentially be replaced with bone after a period of time, it could retain the short-term biological advantages of hydroxyapatite without the longterm disadvantages. Although little is known about this material in clinical practice, in vivo and in vitro studies have demonstrated that calcium phosphate cement serves as a promising material for grafting applications [1-3]. In our preliminary study, we successfully self-invented a modified absorbable compound bone cement by mixing allogeneic bone powder, $\beta$-tricalcium phosphate $(\beta-\mathrm{TCP})$, and bone powder plus calcium phosphate cement (BCPC) at a fixed proportion [1]. 
Subsequently, we applied this novel bone cement in animal studies. The experimental results have demonstrated that the self-designed BCPC can induce osteogenesis as bone graft, which possesses excellent molding capability and mechanical property. In addition, it rarely generates the heat energy during the process of solidification. The bone graft properly matches with surrounding bone defects, which reduces the risk of the migration and dislocation of bone fragment. Consequently, this material is of potential significance for the repairing of bone defects and severe comminuted bone fracture in clinical settings. Prior to clinical application, the mechanism underlying the effect of BCPC upon inducing the differentiation and proliferation of bone mesenchymal stem cells (BMSCs) should be elucidated. In this in vitro experiment, BMSCs were isolated from the rabbit models and co-cultured with different types of bone grafting materials to evaluate the effect and clarify the underlying mechanism of inducing proliferation and osteogenesis of BMSCs, aiming to provide fundamental evidence for clinical application of this novel material.

\section{Methods}

\section{Materials and reagents}

Conventional calcium phosphate cement (Shanghai Rebone Biomaterials Co., Ltd., Shanghai, China), $\beta$-tricalcium phosphate $(\beta-\mathrm{TCP})$, hyperpure hydroxyapatite powder (HA) (Sigma, Germany), calcium monohydrogen phosphate and calcium carbonate (Xilong Scientific, Shantou, China), and alginic acid sodium (Alfa Aesar, USA). DMEM culture medium (Hyclone, USA), fetal bovine serum (Sigma, Germany), alkaline phosphatase (AKP) kit (Nanjing Jiancheng Bioengineering Institute, China), RNA extraction kit (BIO BASIC INC., USA), DNA synthesis kit and SYBR Green qPCR kit (Fermentas, USA), and BMP2 (Bone Bank of the Forth Military Medical University, China).

\section{Methods \\ Preparation of allogeneic bone powder from rabbit models \\ Fresh cortical bone was collected from the rabbits, and soft tissues were eliminated, cut into pieces with a diam- eter $<1 \mathrm{~cm}$, washed, dehydrated in absolute alcohol for $2 \mathrm{~h}$, soaked in diethyl ether for $1 \mathrm{~h}$, air dried, and stored at $-80{ }^{\circ} \mathrm{C}$. According to the Urist technique, the bone particles were crushed into bone powder, sieved through 60-80 mesh into $200-300-\mu \mathrm{m}$ bone powder, disinfected by radiation exposure, and restored at $-80{ }^{\circ} \mathrm{C}$ for subse- quent use.}

Preparation of novel compound calcium phosphate cement The $\beta$-TCP, calcium monohydrogen phosphate, calcium carbonate, and hydroxyapatite powder were mingled at a ratio of 6.0:3.0:0.5:0.5 and then mixed with allogeneic bone powder at a ratio of 1.0:0.4, supplemented with $0.25 \mathrm{M} \mathrm{Na}_{2} \mathrm{HPO}_{4} / \mathrm{NaH}_{2} \mathrm{PO}_{4}$ buffer solution containing
$0.8 \%$ alginic acid sodium $(\mathrm{pH}=7.4)$, preserved at $37{ }^{\circ} \mathrm{C}$ and $100 \%$ humidity for $72 \mathrm{~h}$ to create solid blocks of $5 \times$ $5 \times 2 \mathrm{~mm}$ in size, washed by distilled water, disinfected twice by ultrasonography for $20 \mathrm{~min}$, and air dried, and BCPC blocks were obtained. Bone morphogenetic protein (BMP) (40 mg/g) was mixed with calcium phosphate cement to make BMP plus BCPC blocks according to the methods above.

\section{Co-culture of rabbit BMSCs and calcium phosphate cement}

Tibial bone marrow was obtained from New Zealand white rabbits and cultured in fresh DMEM culture medium containing 10\% fetal bovine serum; the BMSCs were digested using $0.25 \%$ trypsin solution at a cell confluence of $90 \%$, passaged at a ratio of $1: 3$, and the quantity of BMSCs was counted by using trypan blue staining after the second cell passage. Calcium phosphate cement blocks were placed in 24-well plate, supplemented with complete culture solution, and pre-moistured for $1 \mathrm{~h}$; the culture solution was abandoned, the BMSCs were inoculated into the vector at a concentration of $2 \times 10^{5}$ cells/well and cultured at $37{ }^{\circ} \mathrm{C}$ and $5 \% \mathrm{CO}_{2}$; and the culture solution was changed daily. No vector was used in the control group.

\section{BMSCs morphology}

After cell inoculation, the morphology and growth of BMSCs were observed under inverted microscope. At $6 \mathrm{~h}, 1-, 3-$, and 6 days after cell inoculation, two vectors were collected from the traditional calcium phosphate cement, modified calcium phosphate cement, and modified calcium phosphate cement plus BMP groups; rinsed by phosphate buffer; fixed in $20 \mathrm{~g} / \mathrm{L}$ glutaraldehyde; dehydrated in gradient ethanol; air dried; and prepared for observation under scanning electron microscope.

\section{BMSC proliferation characteristics after surface adhesion}

At $24 \mathrm{~h}$ after cell inoculation, the BMSCs adhering to both the wall and vector from 5 wells (24-well plate) were digested by trypsin solution. The cell quantity in each well was counted and repeated at 1-7 days after co-culture with BMSCs. The cell growth curve was delineated.

\section{Detection of the BMSCs' ability to secrete AKP}

At $1,3,5$, and 7 days after BMSC inoculation, the BMSCs from 5 wells (24-well plate) were digested by $100 \mu \mathrm{L}$ Triton-100 solution containing albuminolysis inhibitor and preserved at $4{ }^{\circ} \mathrm{C}$ overnight. According to the AKP detection kit instructions, the absorbance value was measured at a wavelength of $410 \mathrm{~nm}$. The cell counting was repeatedly performed at 1, 3, 5, and 7 days after BMSC inoculation. The mean absorbance value was calculated from the values of 1000 cells. 


\section{Real-time $P C R$}

At 10 days after co-culture, an equivalent quantity of BMSCs was collected from three wells in each group. Total RNA extraction was carried out. RT-PCR was performed to quantitatively measure the expression levels of aggrecan, collagen I, collagen II, AKP, and OSX; the GAPDH was used as internal control. The difference in the mRNA expression was statistically compared using $2^{-\Delta \Delta C T}$ method [2].

\section{Statistical analysis}

SPSS 19.0 statistical software was utilized for data analysis (SPSS Inc., Chicago, USA). All data were expressed as mean \pm standard deviation (SE). Group comparison was performed by using $t$ test. A $P$ value of less than 0.05 was considered as statistically significant.

\section{Results}

Cell morphology and proliferation characteristics of BMSCs after isolation and passage

Primary culture: the culture solution was half exchanged at $24 \mathrm{~h}$ and fully exchanged at $72 \mathrm{~h}$. The non-adherent cells were eliminated, which were in long or shuttle shape, and gathered in mass (Fig. 1a). The incubation period of cell proliferation was initiated at 4 to 7 days after primary culture. The mitosis became active at 8 days, the cell cloning was enlarged in exponential growth phase at 9 to 14 days, and the cells completely overspread the bottom of the culture flask at 15 days.

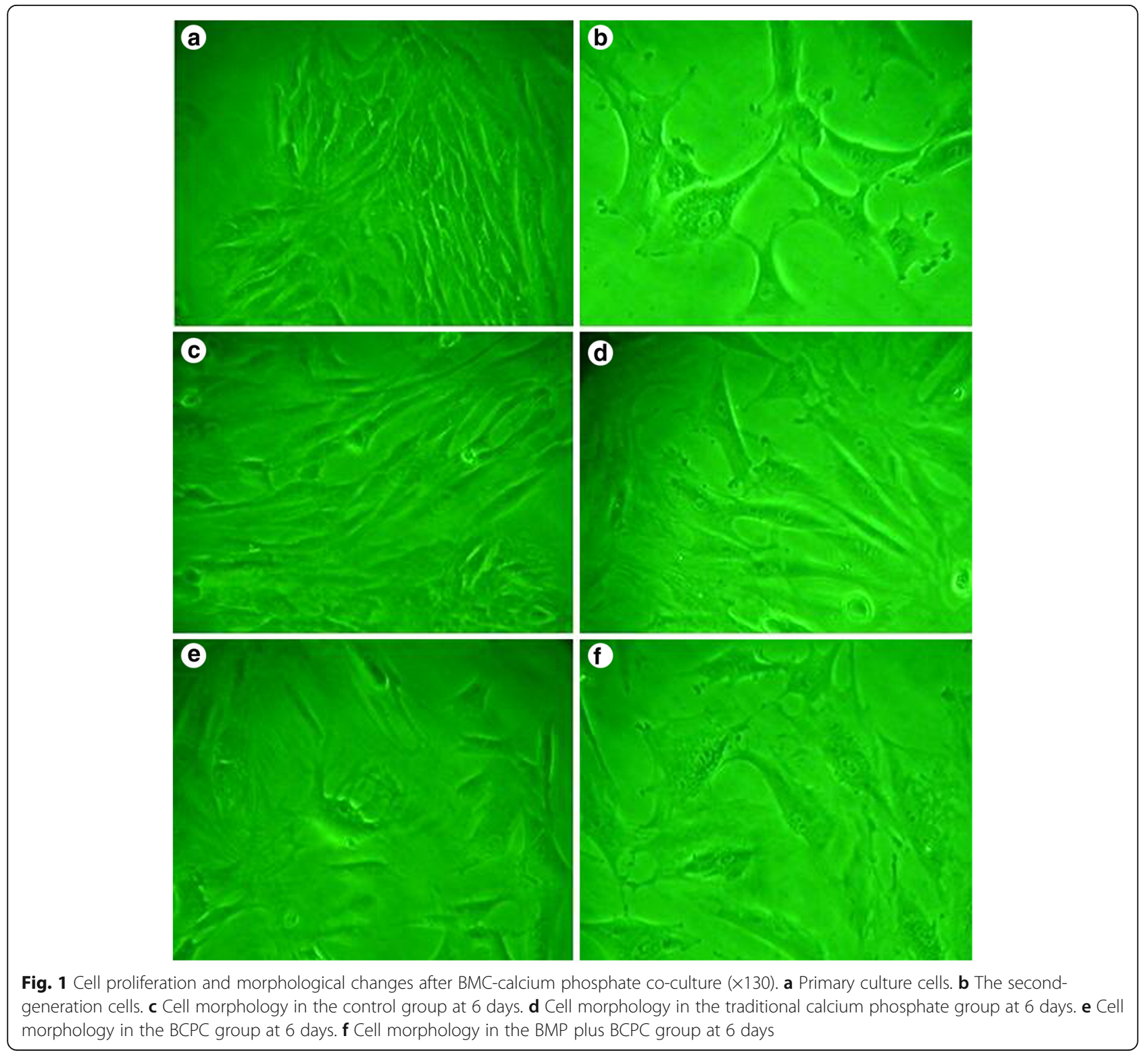


Cell passage: during cell passage, the cells proliferated at a faster speed, attached to the wall at $4 \mathrm{~h}$, and evenly overspread the bottom of culture flask at $7 \mathrm{~h}$. No significant change was observed in cell morphology between primary culture and cell passage (Fig. 1b).

\section{Cell differentiation and proliferation after co-culture of BMSCs and bone cement}

After co-culture, the cells closely adhered to the vector margin. Cellular neurite and pleomorphic cells were observed in the BCPC and BMP plus BCPC groups, whereas shuttle-shaped cells were seen in the traditional calcium phosphate cement and control groups, as illustrated in Fig. 1c, d. After cell co-culture, the cells maintained normal cellular division and proliferation. No statistical significance was noted among different groups (all $P>0.05$ ), as illustrated in Fig. 2.

\section{Cell morphology and ultrastructure}

Compared with traditional calcium phosphate cement, the modified $\mathrm{BCPC}$ had loose structure and large pore size from 200 to $300 \mu \mathrm{m}$, as illustrated in Fig. 3. The BMSCs proliferated on the BCPC, outgrew pseudopod, and invaded into the micropore. At 6 days, the cell quantity was significantly increased and gathered in mass surrounding the micropore. A slight quantity of BMSCs extended into the micropore in a shuttle or polygonal shape. In the traditional calcium phosphate cement group, the superficial cell quantity was low and the cells merely proliferated on the BCPC surface. No evident disparity was observed in cell morphology and distribution, as illustrated Fig. 4.

\section{Detection of AKP activity after cell co-culture}

Along with the prolongation of incubation time, the levels of AKP in the traditional calcium phosphate cement and control groups were slightly increased with no statistical significance (both $P>0.05$ ). At 7 days after cell co-culture, the AKP content in the BCPC group was significantly higher compared with those in the traditional calcium phosphate cement and control groups (both $P<$ 0.05). At 5 and 7 days after cell co-culture, the level of $\mathrm{AKP}$ in the BMP plus BCPC group was significantly higher compared with those in other groups (all $P<0.05$ ), as illustrated in Table 1.

\section{Fluorescent real-time PCR}

At 10 days after cell co-culture, the expression levels of aggrecan, collagen I, collagen II, AKP, osteogenic, or chondrogenic genes in the $\mathrm{BCPC}$ and BMP plus $\mathrm{BCPC}$ groups were significantly upregulated compared with those in the traditional calcium phosphate cement and control groups (all $P<0.05$ ). No statistical significance was documented in the expression levels of these parameters between the traditional calcium phosphate cement and control groups (all $P>0.05$ ), as illustrated in Table 2.

\section{Discussion}

Owing to bone diseases and traumatic events, millions of patients worldwide need to undertake bone grafting operations annually [1]. Bone grafting is the procedure of replacing missing or damaged bones with materials from either the patients (autograft) or donors (allograft). There is an increasing demand for synthetic bone substitutes, which are free from the limitations of bone supply, inconsistency, and disease. Moreover, it is of great potential to utilize these substitutes in conjunction with patients' own cells or recombinant growth factors to accelerate or improve the quality of bone regeneration, which is known as tissue engineering. A wide range of synthetic materials, including metals, ceramics polymers, and cements, have been proposed and developed as bone substitutes. Among them, calcium phosphate cements have been attracting widespread attention due to
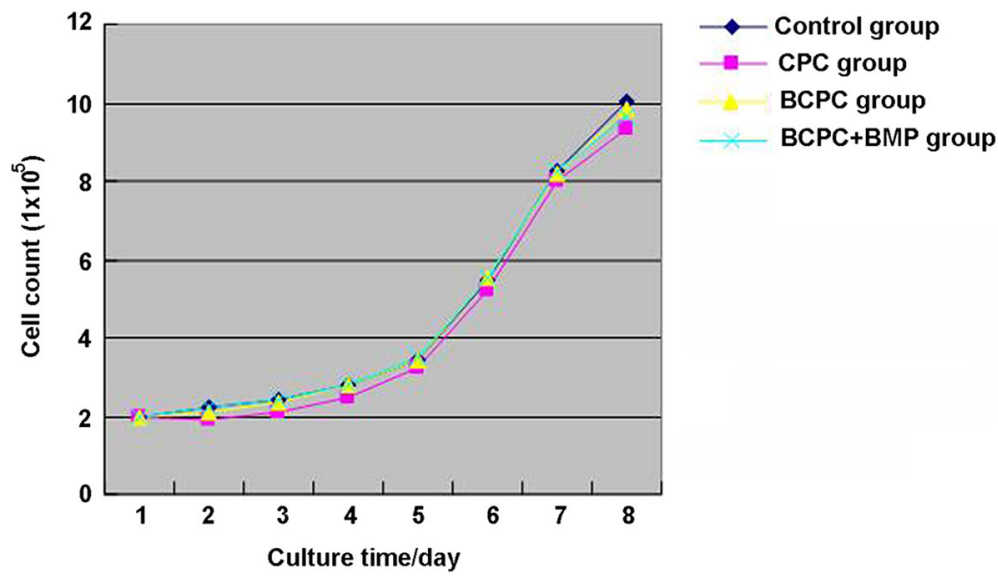

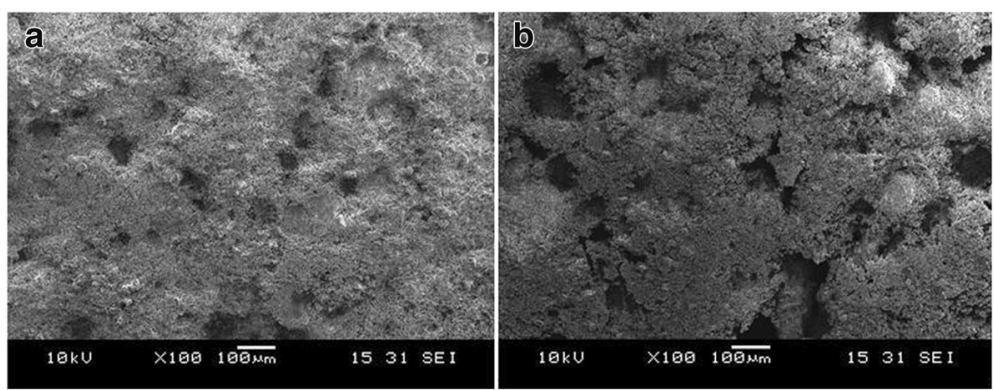

Fig. 3 Morphology of bone cement surface under scanning electron microscope $(\times 100)$. a Traditional calcium phosphate cement group. b BCPC group

their excellent biological behaviors, such as biocompatibility, bioactivity, and osteoconductivity. In addition to their excellent biological behavior, the main advantages of calcium phosphate cements are that they can be injected and have the ability to harden in vivo at body temperature. After mixing of the solid and liquid phases, calcium phosphate cements form a viscous paste, which can be easily manipulated and shaped and injected into a defect area, not only avoiding invasive surgical procedures but also providing intimate adaptation to the surrounding bone even for irregularly shaped cavities, representing a unique advantage over bioceramics, which are difficult to machine and shape. Another important feature of calcium phosphate cements is that they are intrinsically microporous. The micropores are left by extra aqueous solution after hardening of calcium phosphate cements due to intergranular spaces, with pore size in the range of submicro- or micrometers. Such micropores are appropriate for the impregnation of biological fluids into calcium phosphate cements and contribute to the resorption and replacement of calcium phosphate cements by bone tissues. However, due to relatively weak cohesion, calcium phosphate cements are likely to disintegrate upon early contact with blood or biological fluids. Another main challenge encountering calcium phosphate cements is that they possess poor mechanical properties in terms of strength
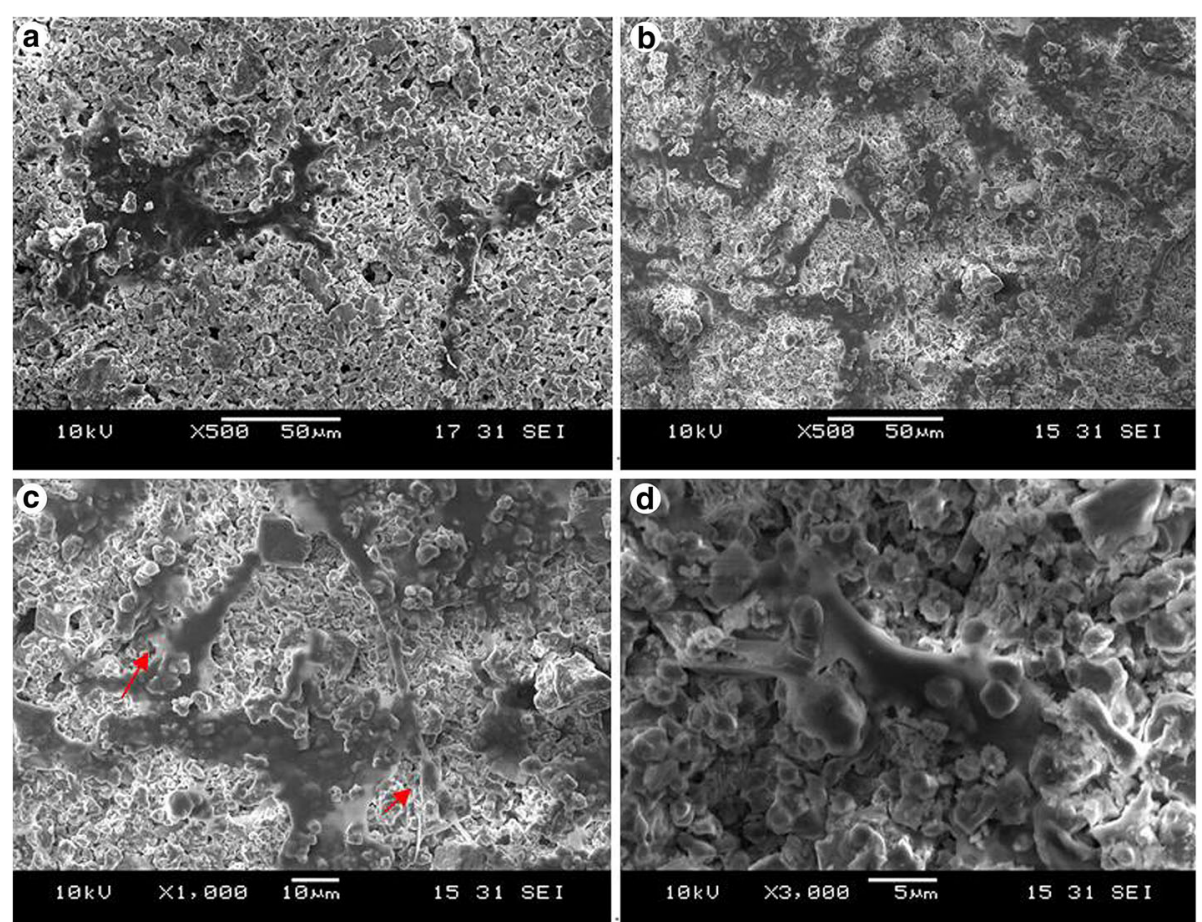

Fig. 4 Observation of growth status after BMC-BCPC co-culture at 5 day under scanning electron microscope. a At 1 day, the cell quantity was small, which were scattered on the bone cement surface $(\times 500)$. b At 5 days, the cell quantity was significantly increased and gathered in mass on the surface $(\times 500)$. $\mathbf{c}$, d The cell pseudopod invaded into the micropore $(\mathbf{c}, \times 1000)$, and the cells grew into the material gap (d, $\times 3000)$ 
Table 1 Changes in AKP activity after cell co-culture $(x \pm s, n=5)$

\begin{tabular}{|c|c|c|c|c|}
\hline \multirow[t]{2}{*}{ Group } & \multicolumn{4}{|l|}{ Culture time $(\mathrm{d})$} \\
\hline & 1 & 3 & 5 & 7 \\
\hline TCP & $0.00614 \pm 0.00038$ & $0.00609 \pm 0.00034$ & $0.00633 \pm 0.00029$ & $0.00679 \pm 0.00041$ \\
\hline $\mathrm{BCPC}$ & $0.00598 \pm 0.00026$ & $0.00617 \pm 0.00031$ & $0.00680 \pm 0.00038$ & $0.00787 \pm 0.00038$ \\
\hline $\mathrm{BCPC}+\mathrm{BMP}$ & $0.00600 \pm 0.00037$ & $0.00637 \pm 0.00042$ & $0.00754 \pm 0.00051$ & $0.00992 \pm 0.00037$ \\
\hline Control & $0.00613 \pm 0.00029$ & $0.00631 \pm 0.00045$ & $0.00640 \pm 0.00036$ & $0.00690 \pm 0.00028$ \\
\hline
\end{tabular}

toughness, brittleness, and reliability, limiting their application in clinical settings $[3,4]$.

To resolve these weaknesses, we modified the composition of calcium phosphate cement and mingled allogeneic bone powder with calcium phosphate cement to create novel BCPC. In our preliminary animal experiment, BCPC was proven to act as an artificial bone graft material. The present investigation was designed to evaluate the osteogenic induction effect of BCPC by mixture and co-culture of rabbit BMSCs and BCPC in vitro. Meantime, traditional calcium phosphate cement was also used as the control material. The experimental outcomes have demonstrated that the modified $\mathrm{BCPC}$ has loose structure and large pore size above $100 \mu \mathrm{m}$ compared with the traditional calcium phosphate cement. Therefore, it creates an excellent microenvironment for cellular and vascular proliferation

Table 2 Expression levels of osteogenic or chondrogenic genes after cell co-culture $\left(2^{-\Delta C T}\right.$ method)

\begin{tabular}{llll}
\hline Target genes & $\begin{array}{l}\text { Avg. } \Delta \mathrm{Ct} \text { (target } \\
\text { gene Ct-GAPDH Ct) }\end{array}$ & $\begin{array}{l}\Delta \Delta \mathrm{Ct} \text { (avg. } \Delta \mathrm{Ct}, \\
\text { target-avg. } \Delta \mathrm{Ct} \text { target gene } \\
\text { control) }\end{array}$ & $\begin{array}{l}\text { amount relative } \\
\text { to control 2 } \Delta \Delta \mathrm{Ct}\end{array}$ \\
\hline Collagen I (a) & $10.98+0.28$ & $0.00+0.28$ & $1.0(0.8-1.2)$ \\
Collagen I (b) & $11.18+0.26$ & $0.20+0.38$ & $0.9(0.7-1.1)$ \\
Collagen I (c) & $10.07+0.17$ & $-0.91+0.33$ & $1.9(1.5-2.3)$ \\
Collagen I (d) & $8.22+0.25$ & $-2.76+0.38$ & $6.8(5.2-8.8)$ \\
Collagen II (a) & $13.15+0.20$ & $0.00+0.20$ & $1.0(0.9-1.1)$ \\
Collagen II (b) & $12.89+0.17$ & $-0.24+0.26$ & $1.2(1.0-1.4)$ \\
Collagen II (c) & $11.58+0.23$ & $-1.57+0.30$ & $3.0(2.4-3.7)$ \\
Collagen II (d) & $10.64+0.18$ & $-2.51+0.27$ & $5.7(4.7-6.9)$ \\
Aggrecan (a) & $10.87+0.19$ & $0.00+0.19$ & $1.0(0.9-1.1)$ \\
Aggrecan (b) & $10.34+0.30$ & $-0.53+0.35$ & $1.4(1.2-1.8)$ \\
Aggrecan (c) & $9.27+0.52$ & $-1.60+0.55$ & $3.0(2.1-4.3)$ \\
Aggrecan (d) & $7.62+0.30$ & $-3.25+0.35$ & $9.5(7.7-11.7)$ \\
AKP (a) & $11.57+0.40$ & $0.00+0.40$ & $1.0(0.8-1.3)$ \\
AKP (b) & $11.70+0.52$ & $0.13+0.65$ & $0.9(0.6-1.4)$ \\
AKP (c) & $9.67+0.25$ & $-1.90+0.47$ & $3.7(2.7-5.1)$ \\
AKP (d) & $8.27+0.28$ & $-3.30+0.49$ & $9.8(7.0-13.8)$ \\
OSX (a) & $14.24+0.26$ & $0.00+0.26$ & $1.0(0.8-1.2)$ \\
OSX (b) & $13.86+0.21$ & $-0.38+0.33$ & $1.3(1.0-1.6)$ \\
OSX (c) & $12.53+0.24$ & $-1.71+0.35$ & $3.3(2.6-4.2)$ \\
OSX (d) & $11.10+0.16$ & $-3.14+0.31$ & $8.8(7.1-10.2)$ \\
\hline
\end{tabular}

[5]. The proliferation rate of BMSCs did not significantly differ among four groups, suggesting that BCPC and BMP impose no evident toxicity upon the BMSCs and yield excellent cellular biocompatibility. At 7 days after cell coculture, the activity of AKP in the BCPC and BMP plus $\mathrm{BCPC}$ groups was significantly higher compared with the traditional calcium phosphate cement and control groups. As a pivotal biomarker of osteoblast differentiation, high AKP activity indicates the high differentiation ability from menchymal stem cells to osteoblasts [6]. RT-PCR demonstrated that the expression levels of aggrecan, collagen I, collagen II, AKP, and osteogenic or chondrogenic genes in the $\mathrm{BCPC}$ and $\mathrm{BMP}$ plus $\mathrm{BCPC}$ groups were significantly upregulated compared with those in the traditional calcium phosphate cement and control groups, especially the $\mathrm{BMP}$ plus $\mathrm{BCPC}$ group, suggesting that $\mathrm{BCPC}$ has certain osteogenic induction activity, which is significantly enhanced after supplement with BMP.

Modified Urist method can properly preserve and release BMP cytokines [7, 8]. BMP is capable of inducing cells with osteogenic potential to differentiate into osteoblasts. Co-culture of BMP from natural extract or gene recombination and vector can repair and accelerate the regeneration of bone tissues [9]. In this investigation, we modified and improved the Urist method by mixing allogeneic bone powder with calcium phosphate cement material to create $\mathrm{BCPC}$, which possesses loose pore structure, which contributes to the proliferation and differentiation of target cells [10]. Previous animal studies have demonstrated that calcium phosphate cement has certain osteogenic induction ability [11-15]. In the present experiment, no significant osteogenic induction activity was observed in the traditional calcium phosphate cement and control groups, probably because the calcium phosphate cement absorbs surrounding active cytokines and possesses certain biological activity. It may also result from the different physicochemical properties of different materials. In this experiment, bone powder rather than the mixture of bone particle and calcium phosphate powder was utilized mainly because compared with bone particle, bone powder favors the release of BMP. The diameter of bone particles is relatively large, which induces injuries to crosslinking structure after hydration response of calcium phosphate, thereby affecting the molding of bone cement. Early filling of 
bone powder functions to support the hydroxyapatite gap following hydration reaction of calcium phosphate cement. Compared with bone particles, bone powder possesses faster degradation and absorption due to smaller diameter, which contributes to subsequent vascular proliferation and invasion. After multiple screening experiments, the optimal ratio of bone powder and calcium phosphate powder is 1:0.4. An insufficient quantity of bone powder affects the biological activity of bone cement. Excessive supplement of bone powder yields bone cement clotting. However, the expression level of collagen III was not quantitatively investigated. In addition, the expression levels of relevant growth factors remain to be elucidated, which contributes to unravel the mechanism underlying the effect of the modified cement. We have supplemented these experiments in our subsequent research.

\section{Conclusions}

Novel compound calcium phosphate cement has proven to possess excellent biocompatibility and osteogenic induction ability. Loose microstructure and large pore size create a favorable environment for BMSCs' proliferation and vessel invasion. It serves as an ideal vector for releasing BMP cytokines, thereby inducing and mediating the differentiation and osteogenesis of BMSCs.

\section{Abbreviations}

AKP: Alkaline phosphatase; BCPC: Bone powder plus calcium phosphate cement; BMP: Bone morphogenetic protein; BMSCs: Bone mesenchymal stem cells; SE: Standard deviation; $\beta$-TCP: $\beta$-tricalcium phosphate

\section{Acknowledgements}

Not applicable.

\section{Funding}

This study was funded by Shantou University Medical College Clinical Research Enhancement Initiative; Shantou City Science and Technology Plan Major Project, Guangdong Province, China (No. 20140305) and Guangdong Science and Technology Plan Project (No. 2008B080701033).

Availability of data and materials

Not applicable.

\section{Authors' contributions}

JZ completed the literature research, clinical studies, experimental studies, and data acquisition. He made the most contribution to manuscript preparation and manuscript editing. JL was a major contributor in the data analysis and definition of intellectual content. GY finished the data analysis and study design. KK was responsible for manuscript review and experimental studies. XW completed the study concepts and study design. He was the guarantor of integrity of the entire study. All authors read and approved the final manuscript.

\section{Competing interests}

The authors declare that they have no competing interests.

\section{Consent for publication}

Not applicable.

Ethics approval and consent to participate

Not applicable.

\section{Publisher's Note}

Springer Nature remains neutral with regard to jurisdictional claims in published maps and institutional affiliations.

\section{Author details}

${ }^{1}$ Department of Spine Surgery, The Second Affiliated Hospital to Shantou University Medical College, The DongXia North Road, Shantou 515041, Guangdong, China. ${ }^{2}$ The Traumatology \& Orthopedics Department, The People's Hospital Affiliated to Fujian University of Traditional Chinese Medicine, Fuzhou, China.

Received: 23 February 2017 Accepted: 17 June 2017

Published online: 29 June 2017

\section{References}

1. Weitao Y, Kangmei K, Xinjia W, Weili Q. Bone regeneration using an injectable calcium phosphate/autologous iliac crest bone composites for segmental ulnar defects in rabbits. J Mater Sci Mater Med. 2008;19:2485-92.

2. Livak KJ, Schmittgen TD. Analysis of relative gene expression data using real-time quantitative $P C R$ and the 2(-delta delta $C(T)$ ) method. Methods. 2001;25:402-8.

3. Fernández E, Vlad MD, Gel MM, López J, Torres R, Cauich JV, et al. Modulation of porosity in apatitic cements by the use of alpha-tricalcium phosphatecalcium sulphatedihy dratemixtures. Biomaterials. 2005:26:3395-404.

4. Theiss F, Apelt D, Brand B, Kutter A, Zlinszky K, Bohner M, et al. Biocompatibility and resorption of a brushite calcium phosphate cement. Biomaterials. 2005;26: 4383-94.

5. Flatley $\mathrm{T}$, Lynch $\mathrm{K}$, Benson M. Tissue response to implants of calcium phosphate ceremic in the rabbit spine. Clin Orthop. 1983;179:246-50.

6. Martin TJ, Sims NA. Os teoclas t-derived activity in the coupling of bone formation to resorption. Trends Mol Med. 2005;11:76-81.

7. Reddi $A H$, Huggins $C$. Biochemical sequences in the transformation of normal fibroblasts in adolescent rats. Proc Natl Acad Sci USA. 1972:69:1601-5.

8. Grosfeld EC, Hoekstra JW, Herber RP, Ulrich DJ, Jansen JA, van den Beucken J. Long-term biological performance of injectable and degradable calcium phosphate cement. Biomed Mater. 2016;12:015009.

9. Axrap A, Wang J, Liu Y, Wang M, Yusuf A. Study on adhesion, proliferation and differentiation of osteoblasts promoted by new absorbable bioactive glass injection in vitro. Eur Rev Med Pharmacol Sci. 2016;20:4677-81.

10. Karageorgiou V, Kaplan D. Porosity of $3 \mathrm{~d}$ biomaterial scaffolds and osteogenesis. Biomaterials. 2005;26:5474-91.

11. Gosain AK, Song L, Riordan P, Amarante MT, Nagy PG, Wilson CR, et al. A 1year study of osteoinduction in hydroxyapatite-derived biomaterials in an adult sheep model: part I. Plast Reconstr Surg. 2002;109:619-30.

12. Yuan H, Li Y, de Bruijn JD, de Groot K, Zhang X. Tissue responses of calcium phosphate cement: a study in dogs. Biomaterials. 2000:21:1283-90.

13. Renno AC, Nejadnik MR, van de Watering FC, Crovace MC, Zanotto ED, Hoefnagels JP, et al. Incorporation of bioactive glass in calcium phosphate cement: material characterization and in vitro degradation. J Biomed Mater Res A. 2013;101:2365-73.

14. Ginebra MP, Canal C, Espanol M, de Groot K, Zhang X. Calcium phosphate cements as drug delivery materials. Adv Drug Deliv Rev. 2012;64:1090-110.

15. Hoppe A, Guldal NS, Boccaccini AR. A review of the biological response to ionic dissolution products from bioactive glasses and glass-ceramics. Biomaterials. 2011:32:2757-74.

Submit your next manuscript to BioMed Central and we will help you at every step:

- We accept pre-submission inquiries

- Our selector tool helps you to find the most relevant journal

- We provide round the clock customer support

- Convenient online submission

- Thorough peer review

- Inclusion in PubMed and all major indexing services

- Maximum visibility for your research 